\title{
On the Influence of Internal and External Factors on the Processes of Corrosion-Mechanical Fracture of High-Strength Low-Alloy Steels
}

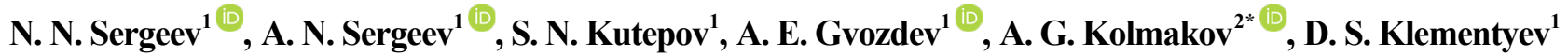 \\ ${ }^{1}$ Tula State Lev Tolstoy Pedagogical University, Lenin Prospekt Tula, Russia \\ ${ }^{2}$ Institution of Russian Academy of Sciences, A. A. Baikov Institute of Metallurgy and Material Science RAS, Leninsky Prospekt \\ Moscow, Russia \\ E-mail: imetranlab10@mail.ru
}

Received: 27 November 2019; Revised: 18 March 2020; Accepted: 21 March 2020

\begin{abstract}
The article studies the influence of internal and external factors on the process of corrosion-mechanical fracture. It is shown that depending on the conditions of hydrogen charging (temperature and chemical composition of the corrosive medium, type of polarization), the mechanical properties of low-alloy steels change dramatically during the initial period of the experiment. With further increase in the holding time in a corrosive medium without polarization, there is no significant change in mechanical properties. It was found that an increase in the level of applied tensile stresses leads to a reduction in the incubation period of microcracks during hydrogen cracking. It was found that the sensitivity of low alloy steels to cracking is largely determined by the relaxation ability of the structure-the relaxation of residual peak micro-stresses localized at the grain boundaries and substructure boundaries reduces the sensitivity to corrosion-mechanical fracture.
\end{abstract}

Keywords: long-term strength, tensile stresses, cathodic polarization, corrosive medium, temperature dependences of internal friction

\section{Introduction}

Corrosion-mechanical fracture is a process of discontinuity of the material, manifested in the simultaneous action of corrosive medium and mechanical stress (applied or residual), and is one of the most common types of brittle fracture of high-strength low-alloy steel in chemical, gas and oil, metallurgical, engineering and other industries [1-4].

A special role in the process of corrosion-mechanical fracture of metal and reinforced concrete structures is played by hydrogen interacting with various microdefects of the metal crystal lattice.

The urgency of the issue of stress-corrosion fracture of high-strength low-alloy steels used in the chemical and oilrefining industries, as well as in the reinforcement of reinforced concrete structures caused by the influence of hydrogencontaining media has increased dramatically in recent decades due to the discovery of abnormal effects of hydrogen on the complex properties of metal materials (abnormal plastic auto-fracture of iron, structural-phase transformations, the effect of reversible loss of shape in amorphous metal alloys, and many others) [3, 4]. A significant number of hydrogen sources (corrosion in aqueous solutions, hydrogen absorption during welding operations, application of technological protective coatings or cathodic protection of underground pipelines, etc) causes significant difficulties in describing

Copyright (C2021 A. G. Kolmakov, et al.

DOI: https://doi.org/10.37256/nat.222021959

This is an open-access article distributed under a CC BY license

(Creative Commons Attribution 4.0 International License)

https://creativecommons.org/licenses/by/4.0/ 
the processes of hydrogen destruction of metal materials, such as hydrogen cracking of high-strength low-alloy steels, stress corrosion cracking of stainless steels, cracking of nuclear reactor pipes made of zirconium alloys, hydrogen embrittlement of titanium alloys by forming a hydride, degradation of GaAs monolithic microwave integrated circuits on satellites, etc.

The increased interest in the problem of corrosion-mechanical fracture of metal materials under the action of hydrogen in recent years contributes to understanding the basic laws of the process, but a number of important problems such as the variety of approaches to understanding the micromechanism of cracking using hydrogen, the lack of complex methods that allow taking into account the set of factors that determine the dominant mechanism of destruction during flooding, the lack of a unified database of tests and standardized recommendations for protecting metal structures from flooding at the stages of manufacture, installation and operation remain unresolved.

The greatest tendency to corrosion-mechanical fracture of high-strength low-alloy steels is observed in conditions of long-term static loading at temperatures close to room temperature and is determined by the following main factors [5-11]: the structural state and chemical composition of steel, the level of residual local micro-stresses, the aggressiveness of the corrosive medium, etc. Taking into account these factors is an actual scientific problem related to the variety of approaches to the study of the process of corrosion-mechanical fracture and a small number of standardized methods of corrosion-mechanical tests.

In this regard, there is a need for certification of high-strength low-alloy steels (after manufacture and during the entire period of operation), due to the variability of their properties and specific operating conditions, which are not sufficiently taken into account by manufacturers.

The purpose is to study the effect of the type and temperature of the aggressive medium, the sample holding time, and the level of internal local micro-stresses on the kinetics of the process of corrosion-mechanical fracture of low-alloy steels.

\section{Research materials and methods}

The influence of internal (level of local micro-stresses) and external (temperature and chemical composition of the aggressive medium, holding time, polarization conditions) factors on the process of corrosion-mechanical fracture of low-alloy steels was studied on full-scale samples of steels 20ГC2 (20GS2), 35ГC (35GS), 80C (80C) smooth-rolled and periodic profiles $\varnothing 12$ and $14 \mathrm{~mm}$ and $l=300 \ldots 400 \mathrm{~mm}$ in the delivery state (Figure 1). The chemical composition of low-alloy steels under study is shown in Table 1. The test setup diagram is shown in Figure 2.

Table 1. Chemical composition and type of treatment of the steels under study in accordance with the standards GOST 5781-82 and GOST 10884-94

\begin{tabular}{ccccccccc}
\hline \multirow{2}{*}{ no. $\mathrm{p} / \mathrm{p}$} & Steel grade, D, mm & Type of treatment & \multicolumn{5}{c}{ Content of elements, \% } \\
\cline { 3 - 8 } & & $\mathrm{C}$ & $\mathrm{Mn}$ & $\mathrm{Si}$ & $\mathrm{Ti}$ & $\mathrm{S}$ & $\mathrm{P}$ \\
\hline One & 20ГC2 (20GS2), Ø14 & $\begin{array}{c}\text { High-temperature } \\
\text { thermomechanical } \\
\text { treatment } \\
\text { Two }\end{array}$ & 0.19 & 1.13 & 2.17 & - & 0.040 & 0.016 \\
Three & 35ГC (35GS), Ø14 & $\begin{array}{c}\text { Electrothermal } \\
\text { hardening }\end{array}$ & 0.34 & 1.40 & 0.80 & 0.02 & 0.030 & 0.030 \\
\hline
\end{tabular}




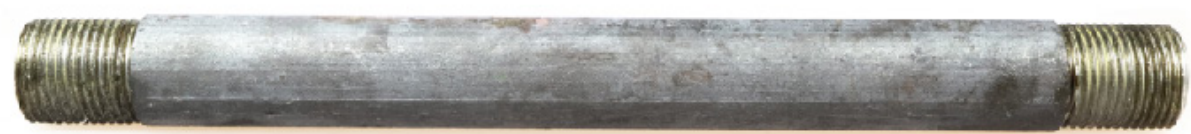

(a)

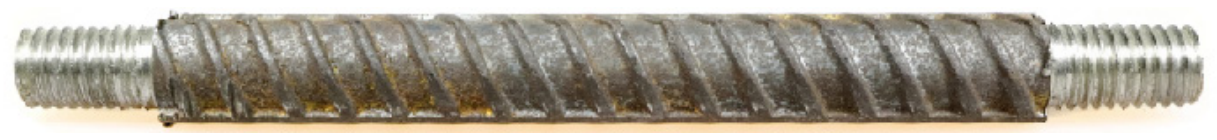

(b)

Figure 1. Full-scale samples for testing for long-term strength in an aggressive medium: (a) smooth-rolled; (b) periodic profile

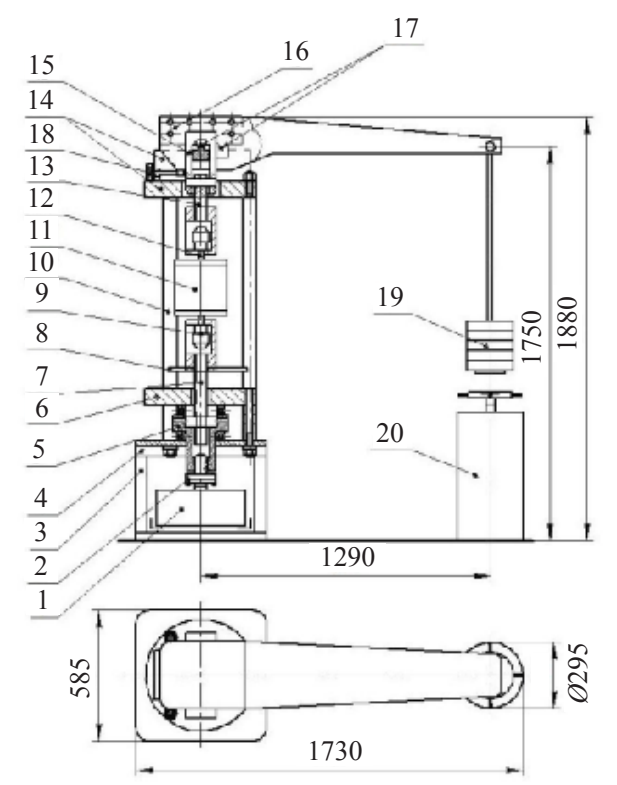

Figure 2. Cell diagram of the installation for long-term strength tests: 1. reducer with an electric motor; 2. coupling; 3. support frame; 4. support plate; 5. nut; 6. lower plate of the power frame; 7. thrust screw; 8. stopper; 9. clamp; 10. rack of the power frame; 11. working vessel; 12. sample; 13. grip; 14. upper plate of the power frame; 15. pull of the upper grip; 16. lever; 17. cargo prisms; 18. scale; 19. loads; 20. stand

The length of the working part of the sample, which was in a special corrosion chamber in contact with an aggressive medium, was $80 \ldots 100 \mathrm{~mm}$. The resistance of steel to corrosion-mechanical fracture was estimated by the time before failure according to the results of tests of $4 . .66$ samples for each point. Graphical dependencies were plotted in the coordinates of the applied stress-time to failure $\left(\sigma_{\ni}-\tau_{\mathrm{P}}\right)$. The material was considered resistant to cracking in hydrogen-containing media under the following conditions [12-16]:

1. for hydrogen cracking-failure does not occur after 200 hours of testing with static tensile stresses of at least $75 \%$ of the critical breaking stress;

2. for stress corrosion cracking-failure does not occur after 100 hours of testing with static tensile stresses of at least $75 \%$ of the critical breaking stress.

Usually, increasing the concentration of acidic media increases the tendency of steel to brittle fracture [1720]. Water solutions of hydrochloric, sulfuric, and other acids with various additives (flood stimulators), as well as solutions containing hydrogen sulfide are used mainly as flood-reducing media [17-20]. To determine the effect of the concentration of electrolyte solutions on the sensitivity of steel to corrosion fracture, samples made of 80C (80C) steel were tested in aqueous solutions of $\mathrm{H}_{2} \mathrm{SO}_{4}$ and $\mathrm{HCl}$ of different concentrations at the same level of tensile stresses $\sigma_{\ni}=0.6$ $\sigma_{\mathrm{B}} \mathrm{MPa}$.

In accordance with a certain pre-fracture time characterizing each stress level, full-scale samples made of steel 
20ГC2 (20GS2) were subjected to a complex and separate influence of various factors in special corrosion chamberscorrosion medium, tensile stresses, cathodic polarization from an external current source at different holding times up to the pre-fracture [17-19].

Also, in the process of holding steel $20 Г \mathrm{C} 2$ (20GS2) in a corrosive medium (in the study of the process of stress corrosion cracking), the temperature of the medium was changed. Then the samples were removed from the installation, washed, dried and cut out samples $l=200 \mathrm{~mm}$ to determine the temperature dependence of internal friction. The time between sample preparation and internal friction measurement did not exceed 1 hour.

Measurements of temperature dependences of internal friction were carried out at different temperatures $\left(20 \ldots 500^{\circ} \mathrm{C}\right)$ at $f \sim 10^{3} \mathrm{c}^{-1}$ according to the resonance technique [21] on full-scale samples of steel 20ГC2 (20GS2), which were cut from rolled steel after aging in a corrosive medium. We also measured the value of the low-temperature background of internal friction $\sim 150^{\circ} \mathrm{C}$, which is associated with the presence of submicropoints in the material. The value of the elastic modulus was determined by the resonant frequency.

The influence of tensile stresses and aggressive medium that causes hydrogen charging on the origin and development of brittle fracture at various stages of testing of 20ГС2 (20GS2) steel was judged by mechanical properties, local and average residual stresses, and the content of hydrogen in the steel. Measurement of residual stresses was carried out by means of X-ray survey of samples on the URS-50IM diffractometer in $\mathrm{CoK}_{\alpha}$ radiation with automatic recording of lines (110) and (220). Micro-stresses were determined by approximation, and macro-stresses were determined by two surveys. Studies were performed on full-scale samples that were previously maintained under voltage in air and in a medium with cathodic polarization for various periods of time. X-ray images taken from the samples allowed tracing the change of stresses of the first kind on the surface after different exposure at the same level of stress $\left(0.5 \sigma_{\mathrm{B}}\right)$ of non-hydrogen-charged and electrolytically hydrogen-charged samples up to fracture.

\section{Results and discussion}

\subsection{Influence of composition and temperature of $\mathrm{H}_{2} \mathrm{SO}_{4}$ and $\mathrm{HCl}$ aqueous solutions}

The type of medium and its concentration affect the long-term strength of low-alloy steels. The study results of the effect of the concentration of $\mathrm{H}_{2} \mathrm{SO}_{4}$ and $\mathrm{HCl}$ aqueous solutions on the sensitivity of $80 \mathrm{C}(80 \mathrm{C})$ steel to corrosion cracking at the same level of tensile stresses $\sigma_{\ni}=0.6 \sigma_{\mathrm{B}} \mathrm{MPa}$ are shown in Figure 1. With an increase in the concentration from 2 to $10 \%$, there is a gradual reduction in the time to failure, and then a slight increase in resistance, which is probably due to changes in this range of concentrations of the rates of hydrogen charging and corrosion of the stressed sample. With a further increase in the concentration, a decrease in resistance is observed, and in $\mathrm{H}_{2} \mathrm{SO}_{4}$ solutions, an increase in the concentration of more than $45 \%$ increases the resistance. Figure 3 shows that steel $80 \mathrm{C}$ (80C) exhibits lower resistance in $\mathrm{HCl}$ solutions compared to $\mathrm{H}_{2} \mathrm{SO}_{4}$ solutions, which can be explained by the specific influence of chlorine anions.

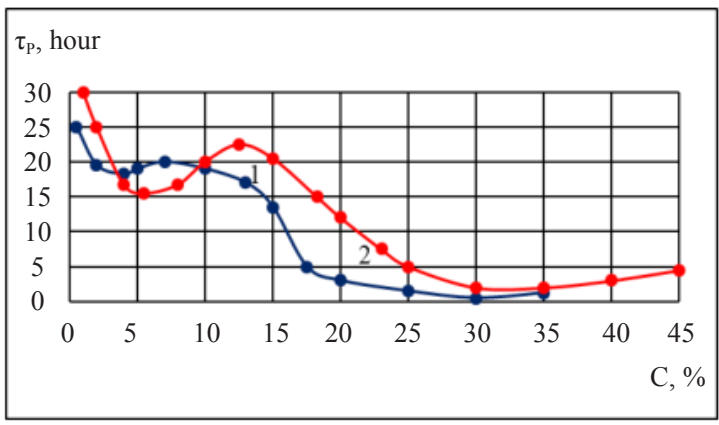

Figure 3. Dependence of time to failure of $80 \mathrm{C}(80 \mathrm{C})$ steel samples on the concentration $(\mathrm{C}, \%)$ of aqueous acid solutions: (1) $\mathrm{HCl}_{\text {; }}(2) \mathrm{H}_{2} \mathrm{SO}_{4}$ 
Figure 4 shows the study results of the effect of the concentration of $\mathrm{H}_{2} \mathrm{SO}_{4}$ solution on the corrosion process rate of the periodic profile $\varnothing 10 \mathrm{~mm}$ of steel 35ГC (35GS). Weight losses of various concentrations were determined after 200 hours of testing. It can be seen that raising the concentration up to $16 \% \mathrm{H}_{2} \mathrm{SO}_{4}$ increases the rate of corrosion; raising the level of tensile stresses also accelerates the process under consideration.

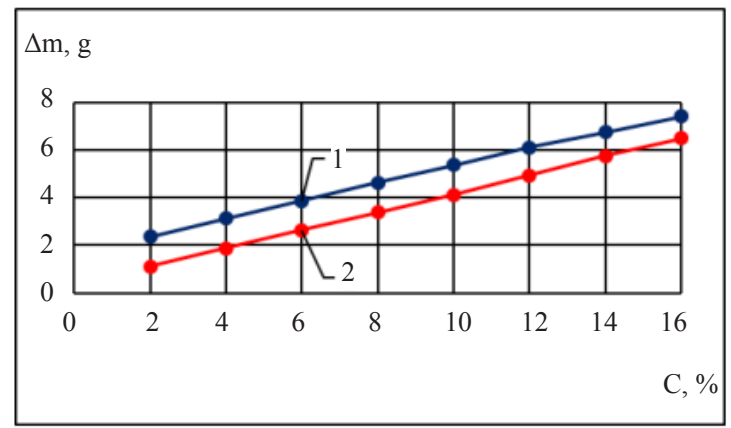

Figure 4. Effect of $\mathrm{H}_{2} \mathrm{SO}_{4}$ solution concentration on weight loss of steel $35 \Gamma \mathrm{C}$ (35GS) (at 200 hours test base) at stresses $\left(\sigma_{\ni}, \mathrm{MPa}\right)$ : (1) $0.7 \sigma_{\mathrm{B}}$; (2) $0.5 \sigma_{\mathrm{B}}$

To clarify the prevailing factor that is responsible for the process of brittle destruction, the method of electrochemical polarization was used. Figure 5. presents the full curve of resistance of low-alloy steel 80C (80C) at anodic and cathodic polarization. The resistance of $80 \mathrm{C}(80 \mathrm{C})$ at a voltage of $600 \mathrm{MPa}$ varies along a domed curve depending on the current density of the cathodic polarization. Thus, with a small increase in the cathode current density, there is a sharp increase in the resistance of low-alloy steel under study, followed by a sharp decrease in it. A sharp increase in the resistance at low cathodic current densities shows that in this corrosive solution, along with hydrogen depolarization, anodic processes are actively occurring, which are suppressed during cathodic polarization. When the cathode current density $D_{\mathrm{K}}=60 \mathrm{~A} / \mathrm{m}^{2}$, there is a decrease in the resistance drop, and when the current density is greater than $300 \mathrm{~A} / \mathrm{m}^{2}$, the resistance does not change much.

Anodic polarization prevents the adsorption of hydrogen on the sample surface, and also smoothens the potential difference caused by stress concentrators, which leads to an increase in resistance. With the subsequent increase in the anode current density, the electrochemical dissolution of the sample is accelerated, which leads to the formation of microcracks and the corrosion cracking under stress. At current densities exceeding $300 \mathrm{~A} / \mathrm{m}^{2}$, the time to failure was virtually unchanged due to the limited ability of the metal surface to absorb hydrogen, as well as due to the formation of a solid jacket of hydrogen bubbles around the cathode that prevented the medium from contacting the sample.

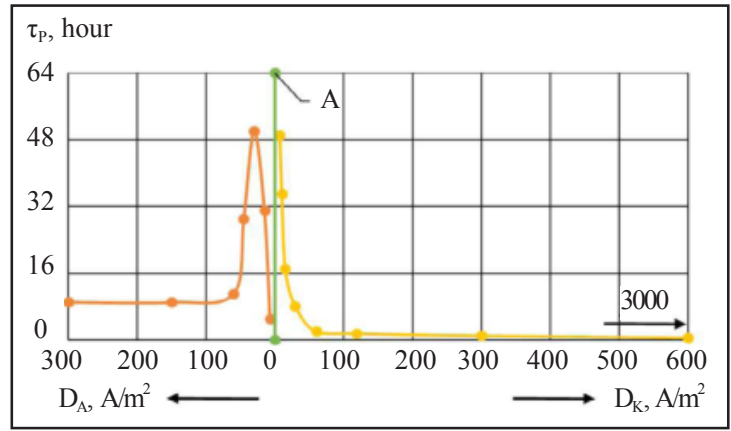

Figure 5. The effect of anodic and cathodic polarization on the brittle fracture of $80 \mathrm{C}(80 \mathrm{C})$ steel at $\sigma_{\ni}=600 \mathrm{MPa}$ in an aqueous solution of $4.5 \% \mathrm{H}_{2} \mathrm{SO}_{4}+2.5 \% \mathrm{NH}_{4} \mathrm{CNS}$; point A corresponds to the stability of samples without polarization 
The results of the study on the effect of changing the temperature of the experiment from $25^{\circ} \mathrm{C}$ to $100^{\circ} \mathrm{C}$ on the long-term strength of $20 \Gamma \mathrm{C} 2$ (20GS2) steel when tested in the accepted experimental corrosion medium are shown in Figure 6. When the level of tensile stresses increases, there is a sharp decrease in the resistance at both room and elevated temperatures. Moreover, when the value of tensile stresses decreases to less than $600 \mathrm{MPa}$, the intersection of these curves is observed and the value of resistance in the medium at a temperature of $100^{\circ} \mathrm{C}$ is slightly higher. This is probably caused by a more intense relaxation of local micro-stresses occurring at $100^{\circ} \mathrm{C}$ than at room temperature and a change in the degree of saturation of the metal with hydrogen. The sharp decrease in the sensitivity of 20ГC2 (20GS2) to hydrogen cracking during 2 hours and a temperature of $20^{\circ} \mathrm{C}$ can be explained by the fact that at the time of the sample's pre-destruction, there is an intense growth of microfractures and their subsequent merging into a main macrofracture, resulting in an avalanche-like destruction of a clear opening of a sample.

Additional tests of low-alloy steel 20ГC2 (20GS2) Ø10 mm after high-temperature thermomechanical treatment with the structure of bainite showed that as the conditions of the experiment are tightened, there is a decrease in the long-term strength, and with a decrease in the level of tensile stresses, the resistance of the studied steel in the accepted corrosion solutions increases.

Analyzing the results obtained, it can be found that when the holding time changes in a corrosive medium, there is a slight change in the strength limit and, to a greater extent, in the yield strength. This can be explained by the course of adsorption phenomena, electrochemical and diffusion processes, which can lead to both hardening and minor plasticization of the sample surface. There is a consistent alternation of hardening and softening processes, which can be caused by local micro-riveting and relaxation of residual stresses. The moment of the greatest softening corresponds to a slight plasticization of the sample, which is reflected in its elongation. Despite the difference in the impact of the media used, the same processes are observed mainly due to a slight change in mechanical properties, probably caused by the creation of additional local micro-stresses in the surface layer and the violation of the continuity of the metal. Increasing the temperature of the experiment accelerates these processes.

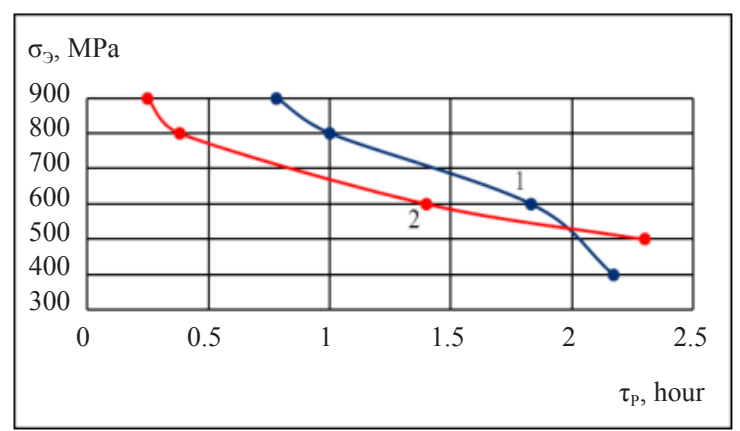

Figure 6. Effect of the experiment temperature on the long-term strength of the steel 20ГC2 (20GS2) in an aqueous solution of $4.5 \% \mathrm{H}_{2} \mathrm{SO}_{4}+2.5 \% \mathrm{NH}_{4} \mathrm{CNS}\left(D_{\mathrm{K}}=60 \mathrm{~A} / \mathrm{m}^{2}\right): 1$ is long-term strength at $25^{\circ} \mathrm{C} ; 2$ is long-term strength at $100^{\circ} \mathrm{C}$

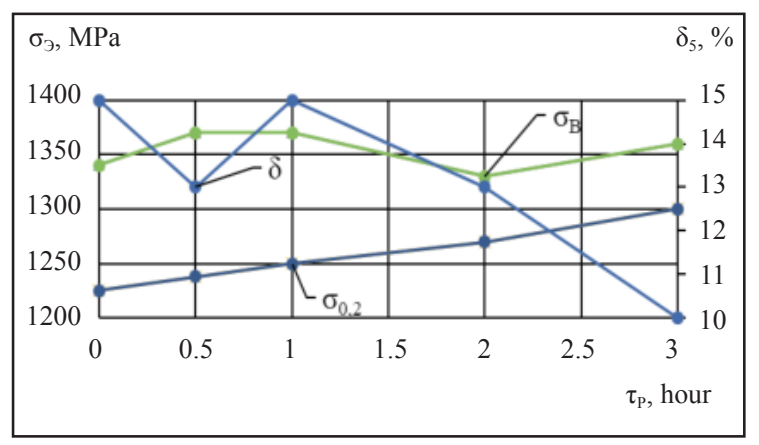

Figure 7. Influence of the duration of exposure on the change of mechanical properties of steel samples 20ГC2 (20GS2) at exposure in aqueous solution $4.5 \% \mathrm{H}_{2} \mathrm{SO}_{4}+2.5 \% \mathrm{NH}_{4} \mathrm{CNS}$ with cathodic polarization $\left(\mathrm{D}_{\mathrm{K}}=60 \mathrm{~A} / \mathrm{m}^{2}\right)$ at room temperature 
To clarify the physical nature of mechanical properties change, the samples were additionally subjected to cathodic polarization in an aqueous solution of $4.5 \% \mathrm{H}_{2} \mathrm{SO}_{4}+2.5 \% \mathrm{NH}_{4} \mathrm{CNS}$ at a current density $\mathrm{D}_{\mathrm{K}}=60 \mathrm{~A} / \mathrm{m}^{2}$ (Figure 7).

The change in mechanical properties $\left(\sigma_{\mathrm{B}}, \sigma_{0.2}\right)$ in the initial time period (up to 0.5 hours) is insignificant, which is explained by the suppression of electrochemical processes on the sample surface due to cathodic polarization. At the same time, a sharp change in the relative elongation of the sample $\delta_{5}$, in the initial time period (up to 0.5 hours) can be explained as follows: due to the interaction of diffusion-mobile hydrogen with dislocation clusters, the latter are blocked, which causes the material to harden, resulting in a decrease in the plasticity characteristics. The increase in plasticity characteristics during the duration of electrolytic hydrogen charging within 0.5-1 hour can be explained by the opening of microcracks, which results in the relaxation of peak micro-stresses and the subsequent increase in the relative elongation. Further electrolytic hydrogen charging (more than 1 hour) leads to even more intensive surface hardening due to hydrogen diffusion and its molization in near-surface defects, creating additional local micro-stresses that lead to changes in the substructure and micro-cleavage, which causes a decrease in $\delta_{5}$, depending on the holding time. The results of the experiments show that the steel changes its mechanical properties slightly when it comes into contact with a corrosive medium.

When a tensile load is applied in a boiling solution of nitrates $60 \% \mathrm{Ca}\left(\mathrm{NO}_{3}\right)_{2}+5 \% \mathrm{NH}_{4} \mathrm{NO}_{3}+35 \% \mathrm{H}_{2} \mathrm{O}$ at $110^{\circ} \mathrm{C}$, a more active change in mechanical properties is observed. It is impossible to say for sure what exactly caused this change and whether it creates microcracks or other defects in the structure. The effect on the stressed metal of media that causes hydrogen cracking in combination with cathodic polarization leads to an even more dramatic change in mechanical properties.

\subsection{Influence of the composition of the medium on the origin of microcracks}

When the sample comes into contact with a corrosive medium, the surface-active elements of the medium are adsorbed, which initiate the process of electrochemical corrosion. Despite the lower strength in the initial state, smoothrolled samples at the same levels of applied stress have a lower sensitivity to cracking (Figure 8). This fact is due to the fact that on the surface of the sample of the periodic profile, there are stress concentrators that contribute to the emergence of a difference in electrode potentials and, as a consequence, the flow of anode and cathode processes. In this case, areas with maximum voltages having lower potentials become anodic, and areas with lower voltages become cathodic.

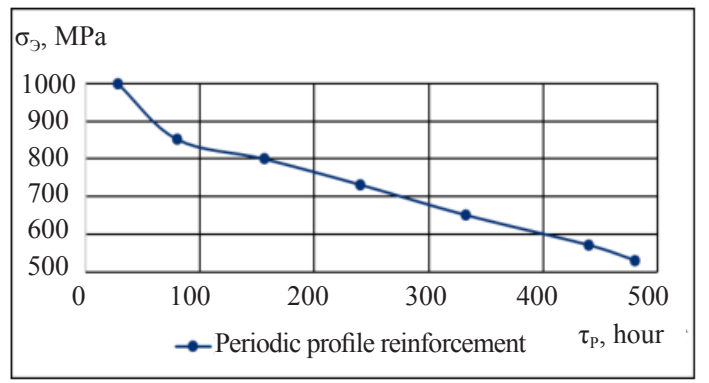

(a)

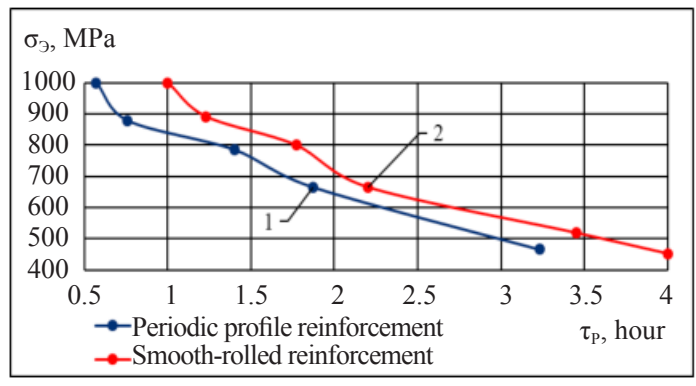

(b)

Figure 8. Effect of corrosive medium (a) and cathodic polarization (b) on the long-term strength of steel 20ГC2 (20GS2) 
When measuring the temperature dependencies of internal friction on samples subjected to pre-exposure in a corrosive medium for different periods of time, a change in the height of the Koester peak was observed, which was associated with a change in the level of micro-stresses in the surface layer of the samples.

\subsubsection{Extract in a solution of nitrates}

The retention of samples up to 2 hours leads to an increase in the level of micro-stresses caused by surface hardening in the initial period, until the formation of "ulcers", which occurs due to the diffusion of elements of the medium into the metal and blocking dislocations. This period corresponds to a change in the modulus, which indicates that both hardening processes and processes associated with discontinuity occur on the surface. Moreover, the intensity of their flow depends on the temperature of the experiment. Drop in the module and increase in the low-temperature background characterizes the period of formation of "ulcers" on the surface. When the exposure time increases, the level of surface micro-stresses stabilizes, followed by a decrease, probably due to the relaxation of these micro-stresses when the "ulcers" are blunted and a slight surface dissolution is observed.

\subsubsection{Exposure in the medium causing hydrogen charging}

During the initial test period ( 0.5 hours), an increase in the height of the Koester peak was observed, which can be explained by a significant increase in micro-stresses, as well as the flow of diffusion processes and the interaction of hydrogen with dislocations, leading to the blocking of the latter. Further hardening occurs with less intensity and is observed up to 2 hours of testing. Then there is a period of softening ( $2 \ldots 10$ hours), as evidenced by the simultaneous decrease in the peak and modulus of elasticity as a result of the formation of defects (ulcers, pores, etc.), which over time can acquire the status of embryonic microcracks. Due to electrochemical dissolution, these microcracks can slowly spread or heal if they become blunted at the top of the crack.

Hydrogen diffusing into the metal increases the level of micro-stresses, the value of which depends on the holding time in a corrosive medium. The growth of micro-stresses alternates with relaxation processes occurring at the time of formation of submicro-cracks. Micro-stresses can reach a certain value, which depends on the intensity of hydrogen charging and the amount of hydrogen absorbed. Consequently, the increase in local stresses in hydrogen-containing media is mainly due to hydrogen charging.

\subsection{Effect of residual tensile stresses on long-term strength}

When working with rod metal structures made of high-strength low-alloy steels, it is necessary to take into account the relationship between applied and residual stresses, since it can lead to an increase or decrease in tensile stresses, i.e., reduce or increase the time to failure. To assess the role of residual stresses, the temperature dependences of internal friction of the initial dry samples subjected to pre-stretching at different stress levels with subsequent exposure were evaluated. The results obtained are shown in Figure 9 (a). In the initial period (holding up to 1 hour), there is a spike in the peak of internal friction (micro-stresses), accompanied by its further decline, which can probably be explained by the course of plastic acts in micro-volumes of metal caused by local overvoltage and ending in relaxation.

With increasing exposure time, there is a slow growth of micro-stresses. The change in the modulus of elasticity, which generally coincides with the change in the level of internal friction, suggests that as a result of fracture hardening, peak stresses are created, under the influence of which submicrotectures may open in the sample volume and on its surface, leading to its softening. When the exposure time is more than 2 hours, there is a slight change in the modulus, which can either increase or decrease, depending on which process prevails the formation of cracks or their healing.

The effect of local micro-stresses on the peak height when testing smooth-rolled samples made of 20ГC2 (20GS2) steel is shown in Figure 9 (b). in the absence of local stress concentrators on the surface, as it should be, the residual micro-stresses in the sample decrease during the fracture. The features of changing micro-stresses are the same as for samples of the periodic profile; only their levels and intensity of relaxation processes differ. 

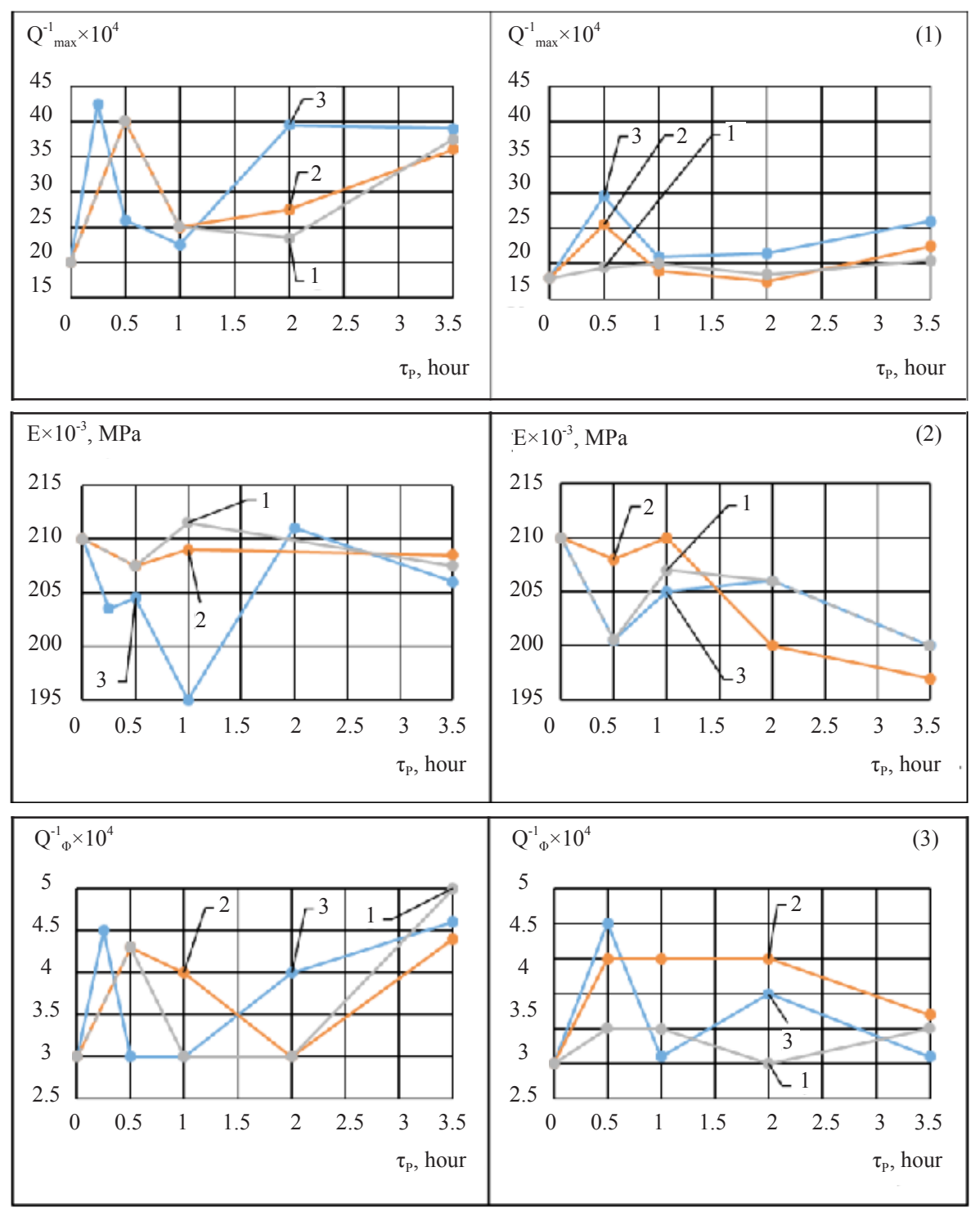

(a)

(b)

Figure 9. Influence of the duration of exposure to air under stress on the height of the Koester peak, the modulus of elasticity and the background $\mathrm{W}$ at $150^{\circ} \mathrm{C}$ of the periodical profile (a) and smooth-rolled rebar (b): (1) $\sigma_{\ni}=0.7 \sigma_{\mathrm{B}} ;(2) \sigma_{\ni}=0.6 \sigma_{\mathrm{B}} ;(3) \sigma_{\ni}=0.5 \sigma_{\mathrm{B}}$

\subsection{The combined effect of the medium and electrolytic hydrogen charging}

Under the combined influence of the medium and electrolytic hydrogen charging, the process of hardening and softening of samples was observed with an increase in the holding time and the repeatability of these processes, but with a smaller voltage drop.

The accelerated flow of these processes is most likely due to the acceleration of the diffusion processes and the molization of hydrogen in the traps, which is expressed in a reduction in the periods of changes in micro-stresses. A sharp drop in the modulus of elasticity at a holding time of more than 2 hours indicates the irreversible nature of hydrogen cracking associated with the formation of discontinuities in the metal. However, the achieved level of microstresses is not sufficient for the development of submicro-cracks and other defects from the existing discontinuities, as a result of which the destruction does not occur. 


\subsubsection{Influence of the medium causing corrosion cracking and tensile stresses}

When testing samples in a corrosive medium under stress, an intensification of corrosion processes was observed. At the same time, the possibility of microfractures is increased by reducing the surface energy and facilitating the release and discharge of dislocations to the metal surface, intensifying electrochemical processes, and diffusing elements of the medium that form solid corrosion products, which can create additional tensile stresses due to the wedging effect. All these factors in the initial period lead to surface hardening, the origin of submicro-cracks and their disclosure. The moment of formation of submicro-cracks is accompanied by relaxation processes that lead to a drop in local microstresses and stabilization of changes in the elastic modulus.

External tensile stresses play a major role in the origin and development of a corrosive microcrack. The presence of a corrosive medium only contributes to faster local processes. The combined effect of external tensile stresses and the corrosive medium leads to an increase in the level of local micro-stresses and a reduction in the time for which these stresses reach a critical value, leading to the origin and further more rapid development of microcracks.

\subsubsection{Influence of the medium leading to hydrogen charging and tensile stresses}

Figure 10 shows the results of gas analysis of steel $20 Г \mathrm{C} 2$ (20GS2) samples after electrolytic hydrogen charging for 1.63 hours at different levels of tensile stresses. This time characterizes the stability of the samples at stresses of 0.5 and $0.6 \sigma_{\mathrm{B}}$. The amount of hydrogen extracted from samples during vacuum heating up to $850^{\circ} \mathrm{C}$ (curve 1), during vacuum melting (curve 2), and the total hydrogen content (curve 3) were measured. During the tests, samples were saturated with hydrogen to varying degrees at different homological stresses. The maximum saturation corresponds to the tests at the applied voltage level $\sigma_{\ni}=0.5 \sigma_{\mathrm{B}}$.

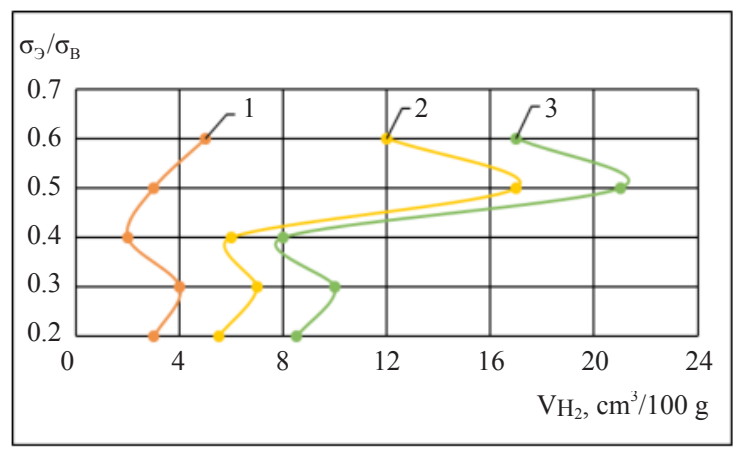

Figure 10. Hydrogen content in steel 20ГC2 (20GS2) samples after hydrogen charging in aqueous solution $4.5 \% \mathrm{H}_{2} \mathrm{SO}_{4}+2.5 \% \mathrm{NH}_{4} \mathrm{CNS}$ a cathodic polarization $\left(\mathrm{D}_{\mathrm{K}}=60 \mathrm{~A} / \mathrm{m}^{2}\right)$ : (1) the hydrogen content in the samples during vacuum heating up to $850^{\circ} \mathrm{C}$; (2) the hydrogen content in the samples during vacuum melting; (3) the total hydrogen content in the samples

The hydrogen charging kinetics can be judged by the results of gas analysis of samples by electrolytic hydrogen charging under a stress of $0.6 \sigma_{\mathrm{B}}$ and $0.4 \sigma_{\mathrm{B}}$ at certain intervals. Increasing the tensile stresses and the time of electrolytic hydrogen charging increases the concentration of hydrogen in the samples to a certain critical level. The hydrogen content at the time of pre-fracture in samples tested under stress of $0.6 \sigma_{\mathrm{B}}$ is $22 \mathrm{~cm}^{3} / 100 \mathrm{~g}$, and at $0.4 \sigma_{\mathrm{B}}-16 \mathrm{~cm}^{3} / 100 \mathrm{~g}$, despite the fact that the duration of electrolytic saturation under a lower stress is almost twice as long. It follows from this that at significant tensile stresses, the defect of the structure increases, which increases the diffusion mobility of hydrogen, and promotes its redistribution, accumulation in local areas, and molization in micro-spaces that pass into microcracks. Increasing the concentration of hydrogen in front of the front of developing microcracks facilitates their development by strengthening the structure (micronaclep). This indicates that the criterion for destruction is not the total content of occluded hydrogen, but its distribution and state.

In local micro-volumes, critical stresses may occur, under the influence of which main microcracks develop. When the latter reach the surface, hydrogen desorption occurs and its concentration drops sharply in the zone of destruction. 
Most of the hydrogen remains in the macrodefects in a molized form and is not extracted at $850^{\circ} \mathrm{C}$. The amount of atomic hydrogen released at this temperature (Figure 10, curve 1) is less dependent on the level of applied stresses. A certain part of hydrogen is captured by defects in the crystal lattice, although its content in the metal volume is almost constant.

In the initial period, there are small compressive stresses on the surface of the initial and enclosed samples, which change their magnitude and sign with increasing duration of exposure under stress. Electrolytic saturation of the sample with hydrogen at first reduces the tensile stresses, and then (after 1.67 hours) brings them to the maximum. A significant pressure of molecular hydrogen in micro volumes causes a sharp increase in tensile stresses; and stress relaxation is caused by the development of microcracks and their merging into main ones.

Reducing the level of tensile stresses can be achieved by desorbing hydrogen from the sample volume and redistributing it (equalizing the concentration by volume). To this end, a low release at $100^{\circ} \mathrm{C}$ for 1 hour was performed before each successive exposure under stress in the medium leading to hydrogen charging, which resulted in a 1.5-fold increase in the stability of the sample.

Judging by the height of the Koester peak and the modulus of elasticity of the initial samples, previously maintained under stress for a given time, local (peak) micro-stresses occur in the steel, which periodically change. Thus, when testing samples in a corrosive medium under stress, an intensification of corrosion processes was observed. The presence of a peak of internal friction indicates that during the initial period of time during hydrogen charging without polarization, hardening processes occur due to fracture and exposure to a corrosive medium.

While aging of the sample in a corrosive medium under tension observed shift of the maximum internal friction to less time that was associated with the accelerated flow of relaxation processes, thereby increasing the possibility of nucleation of microcracks on the surface of the metal and in the surface volume due to the reduction of the surface energy and facilitate the exit of dislocations on the outer surface of the metal and the inner surface of the discontinuities, as well as in result of intensification of electrochemical processes and diffusion of elements, forming a solid corrosion products, which can cause additional tensile stresses due to a wedging effect. All these factors lead to the formation and development of discontinuities in the sample, which is clearly seen by the change in the elastic modulus in the initial period.

However, a further increase in the modulus shows that submicro-cracks formed as a result of hardening caused by the combined action of the corrosive medium and tensile stresses do not develop, and may be prone to healing due to stress relaxation.

As the holding time increases to 100 hours, the level of micro-stresses increases. With a certain holding time, the micro-stresses can reach a value sufficient for the development of the remaining submicro-cracks (mainly in the nearsurface volume, where they are prevented from closing by hydrogen, which diffuses into these areas and as soon as they reach a certain size, crystallizes in them, thereby creating additional tensile stresses due to the increasing pressure of molecular hydrogen). This is confirmed by long-term strength tests.

\subsubsection{Influence of cathodic polarization}

The use of cathodic polarization makes it possible to intensify the process of hydrogen charging. Figure 11 shows that in this case, the tests are accompanied by a sharp decompression. The resulting microcracks reach at least critical sizes, after which their development proceeds spontaneously. Local stresses that occur almost instantly quickly relax due to the formation of a large number of submicro-cracks.

By changing the modulus of elasticity, we can conclude that the first stage (I) is the origin of microcracks. The second stage (II) at stresses $(0.5 \ldots 0.6) \sigma_{\mathrm{B}}$ corresponds to the development of submicro-cracks and proceeds at a lower rate. When the external tensile stresses increase, the duration of the stages increases. The third stage (III) corresponds to the rapid development of the crack due to the fusion of submicro-cracks and microcracks. The duration of this stage is negligible, but in these test conditions is commensurate with the time of origin and development of microcracks. This may be due to the fact that under these test conditions, there is a sharp reduction in the incubation period and the stage of development of microcracks, as well as the origin and development of many disoriented microcracks, which prevents them from merging into the main crack and thereby increases the pre-fracture time. Hydrogen in the process of brittle fracture plays the role of a catalyst for these processes, leading to a sharp decrease in the magnitude of micro-stresses necessary for the promotion of microcracks, thereby facilitating cracking, which is consistent with the data [17-21]. 


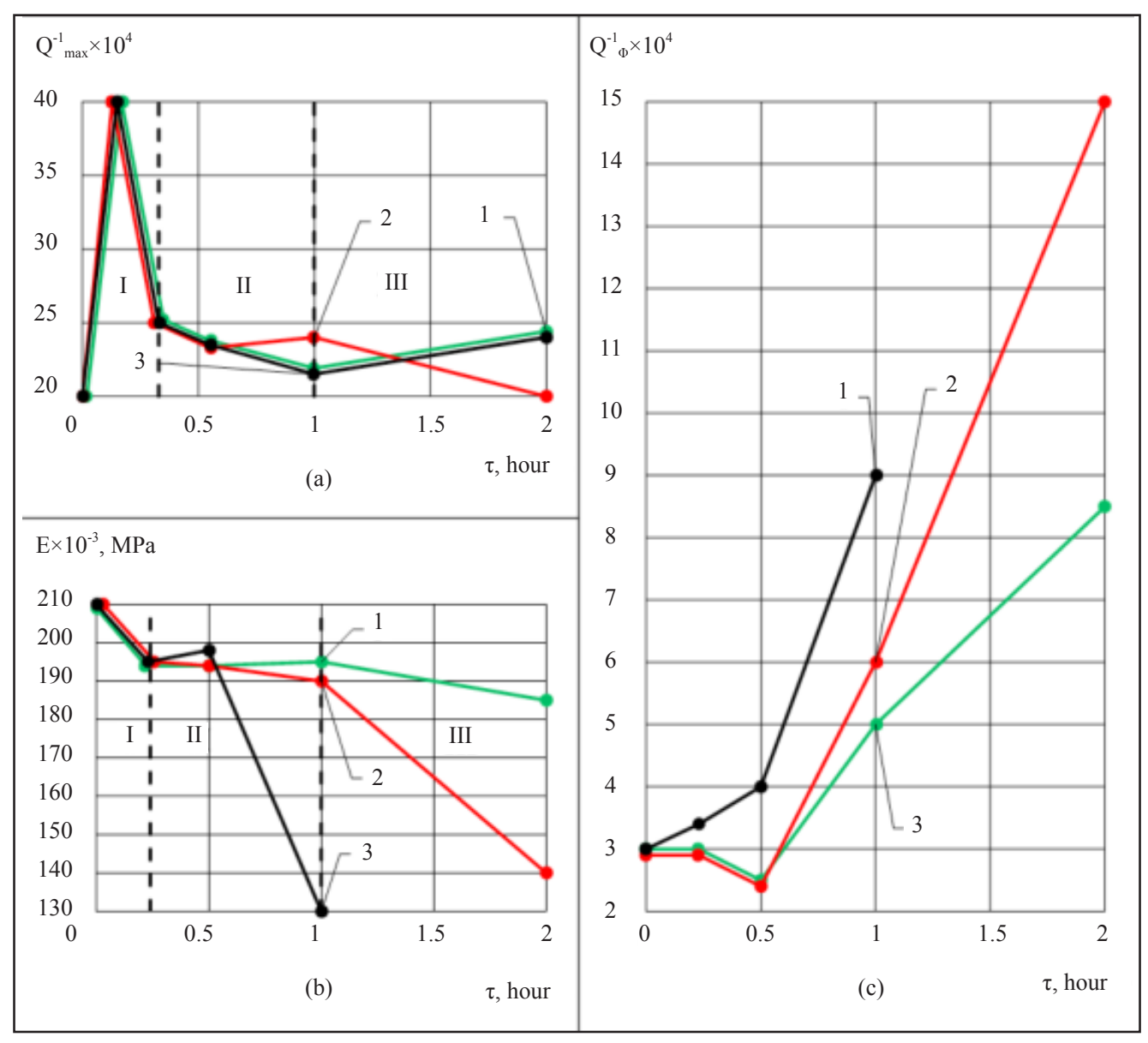

Figure 11. Effect of exposure time in a corrosive medium under stress at $D_{K}=60 \mathrm{~A} / \mathrm{m}^{2}$ on the height of the Koester peak (a), the modulus of elasticity (b) and the background internal friction (c) at $150^{\circ} \mathrm{C}$ : (1) $\sigma_{\ni}=0.7 \sigma_{\mathrm{B}} ;$ (2) $\sigma_{\ni}=0.6 \sigma_{\mathrm{B}} ;(3) \sigma_{\ni}=0.5 \sigma_{\mathrm{B}}$

\section{Conclusions}

1. It is found that the combined effect of tensile stresses (applied and residual) and cathodic polarization on highstrength low-alloy steels $20 Г \mathrm{C} 2$ (20GS2), 35ГC (35GS) and $80 \mathrm{C}(80 \mathrm{C})$ contributes to the intensification of corrosion processes and, as a consequence, to a decrease in long-term strength.

2. It is shown that an increase in the level of applied tensile stresses leads to a reduction in the incubation period of microcracks development during hydrogen cracking. It is likely that the origin and development of cracks in this case occurs mainly in the volume of the sample in places of localization of tensile stresses on the defective parts of the structure and substructure.

3. It is revealed that the long-term strength of high-strength low-alloy steels is largely determined by the relaxation ability of the structure. Relaxation of residual peak micro-stresses localized at grain boundaries and substructure boundaries reduces the sensitivity to corrosion-mechanical fracture.

\section{References}

[1] Azhogin FF. Corrosion cracking and protection of high-strength steels. M.: Metallurgy. 1974; 256.

[2] Petrov LN, Sopronyuk NG. Corrosion-mechanical fracture of metals and alloys. In: OTV. (ed.) Academy of Sciences of Ukraine. R. K. Melekhov. Kiev: Naukova Dumka. 1991. p. 216.

[3] Sergeev NN, Sergeev AN. Hydrogen Embrittlement and Cracking of High-Strength Reinforcing Steel: Monograph. 
Tula: Tulsu publishing house; 2017. p. 180

[4] Chuchelov MV. Theory and Practice of Stress Corrosion Cracking Control on Main Gas Pipelines: Monograph. M.: MAX Press; 2016. p. 336.

[5] Sergeev NN, Ageev VS, Belobragin YA. Hydrogen embrittlement of reinforcing steel 20GS2 during tests for longterm strength. Physical and Chemical Mechanics of Materials. 1981; 1: 20-23.

[6] Tsyrul'nyk OT, Bassarab AI. Structural sensitivity of steels to corrosion-mechanical fracture under conditions of fatigue and cavitation. Materials Science. 1999; 35(4): 581-586. Available from: https://doi.org/10.1007/ BF02365757.

[7] Ioffe AV, Tetyueva TV, Vyboishchik MA, et al. Corrosion-mechanical fracture of tubing from carbon and alloy steels operating in environments containing hydrogen sulfide. Metal Science and Heat Treatment. 2013; 54(9-10): 492-497. https://doi.org/10.1007/s11041-013-9537-4.

[8] Contreras A, Hernández SL, Orozco-Cruz R, et al. Mechanical and environmental effects on stress corrosion cracking of low carbon pipeline steel in a soil solution. Materials \& Design. 2012; 35: 281-289. Available from: https://doi.org/10.1016/j.matdes.2011.09.011.

[9] Kramar V, Veronika DV, Rodkina A, et al. Influence of stress-corrosion fractures on potential of ship-building metals in the sea water. Procedia Engineering. 2015; 100: 1068-1074. Available from: https://doi.org/10.1016/ j.proeng.2015.01.468.

[10] Sergeev NN, Kutepov SN, Sergeev AN, et al. Long-term strength of reinforcing steel 22X2G2A when testing for corrosion cracking in a boiling solution of nitrates. Deformation and Fracture of Materials. 2019; 8: 33-39. Available from: DOI: 10.31044/1814-4632-2019-8-33-39.

[11] Shehovcov A. Influence of internal and external factors on the slow brittle fracture of steel. Fundamental Research. 2013; 11(9): 1841-1845.

[12] GOST R 9.915-2010. Metals, alloys, coatings and products: Test methods for hydrogen embrittlement. M.: STANDARTINFORM. 2011. p. 36.

[13] ASTM F519-17. Standard Test Method for Mechanical Hydrogen Embrittlement Evaluation of Plating/ Coating Processes and Service Environments/in: Annual Book of ASTM Standards. ASTM International, West Conshohocken, PA, USA; 2017.

[14] GOST 9.901.1-89. Unified system of protection against corrosion and aging. Metals and alloys. General Requirements for Test Methods for Stress Corrosion Cracking. M.: Standards publishing house; 1993. p. 21.

[15] GOST 9.901.4-89. Unified system of protection against corrosion and aging. Metals and alloys. Tests for Corrosion Cracking of Samples Under Uniaxial Tension. M.: Standards publishing house; 1993. p. 7.

[16] GOST 9.903-81. Unified system of protection against corrosion and aging. High-strength steels and alloys. Methods of Accelerated Corrosion Cracking Tests. M.: Standards publishing house. 1993. p. 16.

[17] Sergeev NN, Tereshin VA, Chukanov AN, et al. Formation of plastic zones near spherical cavity in hardened lowcarbon steels under conditions of hydrogen stress corrosion. Inorganic Materials: Applied Research. 2018; 9(4): 663-669. Available from: DOI: 10.1134/S2075113318040354.

[18] Sergeev NN, Sergeev AN, Kutepov SN, et al. Mechanism of the hydrogen cracking of metals and alloys, Part I (Review). Inorganic Materials: Applied Research. 2019; 10(1): 24-31. Available from: DOI: 10.1134/ S207511331901026X.

[19] Sergeev NN, Sergeev AN, Kutepov SN, et al. Mechanism of the hydrogen cracking of metals and alloys, Part II (Review). Inorganic Materials: Applied Research. 2019; 10(1): 32-41. Available from: DOI: 10.1134/ S2075113319010271.

[20] Shmakov AA, Singh RN, Matvienko YG, Kolmakov AG. Estimation of the delayed hydride cracking resistance of the pressure tubes in nuclear reactors. Russian Metallurgy (Metally). 2018; 4: 397-401. Available from: DOI: $10.1134 / \mathrm{S} 0036029518040146$.

[21] GOST 25156-82. Metals. Dynamic method for determining elasticity characteristics. M.: Publishing house of standards. 1982. p. 21. 